\title{
Límites de Deuda y Crecimiento Endógeno*
}

\author{
Beatriz de Blas \\ Universidad Autónoma de Madrid \\ beatriz.deblas@uam.es
}

\begin{abstract}
Resumen
Este artículo estudia las consecuencias sobre el crecimiento y el bienestar de diversas políticas fiscales con y sin límites a la deuda pública. En la economía modelo, el gasto público puede tener dos papeles diferentes, bien como un factor en la función de producción, o bien suministrando servicios directamente en la función de utilidad. Cuando hay límites a la deuda, mayores impuestos sobre la renta laboral tienen un efecto positivo sobre el crecimiento si el gasto público es productivo. Lo contrario ocurre cuando es el capital privado el motor del crecimiento. Respecto al bienestar, reducir la deuda recurriendo a mayores impuestos conlleva un menor coste en bienestar que si se hace reduciendo el gasto público cuando éste es productivo.

Palabras clave: crecimiento endógeno, límites de deuda, política fiscal, dinámica transicional.

JEL classification: E62, H3, H5, H63, 041

* Gracias a Jordi Caballé, Jim Costain, Begoña Domínguez, Andrés Erosa, Franck Portier, Michael Reiter, Hugo Rodríguez, y Javier Vallés por sus comentarios. Agradezco la financiación de las «Beques predoctorals per a la formació de personal investigador (FI/FIAP)» de la UAB para el desarrollo de este trabajo. Todos los errores son responsabilidad única del autor.

** Departamento de Análisis Económico: Teoría Económica e Historia Económica, Universidad Autónoma de Madrid, 28049 Cantoblanco (Madrid), España.
\end{abstract}




\section{INTRODUCCIÓN}

Los efectos de la emisión de deuda pública en modelos de crecimiento se han analizado tradicionalmente imponiendo condiciones de no Ponzi sobre el comportamiento limite de la deuda. Sin embargo, se ha prestado poca atención al uso de restricciones más estrictas sobre el préstamo público. Recientemente, este tema ha recobrado interés debido a los límites sobre déficit y deuda pública impuestos sobre los países de la UEM por el Pacto de Estabilidad y Crecimiento.

En este artículo se analizan los efectos de la política fiscal sobre el crecimiento y el bienestar cuando hay límites a la deuda pública. En la economía analizada, el gasto público puede desempeñar dos papeles distintos, bien como un factor más en la función de producción, o bien suministrando servicios directamente en la función de utilidad. El objetivo es estudiar los efectos de diversas políticas fiscales (cambios en los impuestos y en el tamaño del sector público) tanto en el largo plazo como a lo largo de la dinámica transicional.

Una parte de la literatura investiga las consecuencias de la llamada hipótesis de la disciplina de los mercados de crédito ${ }^{1}$. Según esta hipótesis, el comportamiento de los individuos en los mercados de crédito puede restringir el préstamo al sector público. Más concretamente, los agentes privados pueden exigir una prima de riesgo que podría ser creciente en la cuantía de la deuda pública pendiente. La capacidad del gobierno de pagar estas primas determinará su acceso a la financiación del sector privado.

Otro grupo de trabajos se centra en los efectos de imponer exógenamente límites a la deuda. Uctum y Wickens (1997) examinan desde un punto de vista empírico, los efectos de imponer techos a la deuda pública en la restricción presupuestaria intertemporal del gobierno para EEUU y UE desde 1970. Los resultados muestran que la política fiscal llevada a cabo por los gobiernos no es sostenible para la mayoría de los países industrializados en un horizonte infinito, pero es sostenible en el medio plazo en la ausencia de límites. Por otro lado, Chari y Kehoe (1998) analizan la necesidad de restricciones fiscales en la creación de uniones monetarias, especialmente en el caso de la Unión Monetaria Europea. En un modelo estándar con gobiernos benevolentes, estos autores encuentran deseable imponer restricciones fiscales siempre que

\footnotetext{
${ }^{1}$ Véase por ejemplo Bayoumi, Goldstein y Woglom (1995).
} 
la autoridad monetaria no pueda comprometerse a políticas futuras. Ninguno de estos artículos se centra en los efectos sobre el crecimiento. Sin embargo, si el gasto público afecta al crecimiento de la economía, y se financia parcialmente con la emisión de deuda, es importante investigar las consecuencias de limitar esta fuente de financiación.

El modelo desarrollado aquí anida los modelos de crecimiento endógeno de Barro (1990) y de Romer (1986). En el primer caso, se introduce el gasto público en la función de producción, afectando tanto a la productividad del trabajo como a la del capital, y generando crecimiento endógeno. En el segundo caso, el gasto público entra en la función de utilidad de los hogares y el crecimiento endógeno se genera por una externalidad relacionada con aprendizaje por la práctica.

Los resultados muestran que a largo plazo aumentar el impuesto sobre el trabajo tiene un efecto positivo sobre el crecimiento bajo límites a la deuda y si el gasto público es productivo. Sin embargo, cuando el crecimiento es vía capital privado, mayores impuestos sobre la renta laboral sólo reducen el incentivo a trabajar, con un efecto negativo sobre la tasa de crecimiento. Reducir el gasto público tiene efectos negativos sobre el crecimiento si el gasto público es productivo, pero tiene efectos mínimos cuando el consumo público sólo afecta a la utilidad, en ambos casos independientemente de la presencia de un límite a la deuda.

Comparado con la senda de crecimiento equilibrado inicial, aumentar los impuestos para reducir la deuda lleva a la economía a una nueva senda con un mayor crecimiento y menores impuestos, cuando el gasto público es productivo. Del mismo modo, una política fiscal consistente en reducir el tamaño del sector público tiene los efectos contrarios, reduciendo el crecimiento y la producción. Respecto al bienestar, si el gobierno debe alcanzar un menor límite a la deuda, mayores impuestos sobre la renta conllevan un menor coste en términos de bienestar que reducir el gasto público.

El resto del artículo está organizado como sigue. Las Secciones 2, 3 y 4 describen la economía modelo, el equilibrio competitivo y senda de crecimiento equilibrado, respectivamente. La Sección 5 cubre la calibración del modelo. El largo plazo se analiza en la Sección 6, y en la Sección 7 la dinámica transicional. La Sección 8 contiene el análisis del bienestar. Por último, la conclusiones cierran el artículo. 


\section{El MOdelo}

La economía está formada por tres tipos de agentes: hogares, empresas y gobierno. El tamaño de la población está normalizado a uno, por lo que las variables están denominadas en términos per cápita. En esta economía, los individuos toman como dadas las políticas fiscales a la hora de hacer sus decisiones.

Como mencioné anteriormente, el modelo anida dos casos diferentes, cada uno generando distintas externalidades. En primer lugar, las externalidades surgen del papel productivo del gasto público en la función de producción `a-la-Barro (1990); en el segundo caso, el crecimiento endógeno se debe a externalidades en el conocimiento y el aprendizaje por la práctica en la función de producción ‘a-la-Romer (1986). En este último caso, se supone que el gasto público sólo provee de servicios a los individuos en la función de utilidad.

\subsection{Hogares}

La economía consta de un gran número de individuos idénticos que viven para siempre. Los agentes están dotados con una unidad de tiempo que dividen entre ocio, $x(t)$, y trabajo, $l(t)$ : Los hogares consumen un bien homogéneo cuyo precio está tomado como numerario y normalizado a uno. Los individuos obtienen utilidad del ocio, de consumir bienes privados y de los servicios públicos en el caso en que el gasto del gobierno entra en la función de utilidad. La función de utilidad $U[c(t), x(t) ; g(t)]$ es CES de la forma

$$
U[c(t), x(t), g(t)]=\frac{\left[c(t)^{\theta} x(t)^{1-\theta}\right]^{1-\sigma}+\eta\left[g(t)^{\psi}\right]^{1-\sigma}}{1-\sigma}
$$

donde $c(t)$ es consumo; $x(t)$ es la proporción de tiempo dedicada a ocio; $g(t)$ es gasto público; $\sigma>0$ es la inversa de la elasticidad de sustitución intertemporal; $\theta \in[0,1]$ refleja las preferencias del individuo entre consumo y ocio, y $\psi>0$ es un parámetro que mide el impacto de $g(t)$ en el bienestar del individuo. Además, debe cumplirse que $-\infty<1-\sigma<\frac{1}{1+\psi}$, y $\psi(1-\sigma)<1$; para tener utilidad $\operatorname{acotada}^{2}$. Por último,

\footnotetext{
${ }^{2}$ Para el caso de utilidad isoelástica, el parámetro $\psi$ también se puede interpretar como la
} 
el parámetro $\eta=\{0,1\}$ permite analizar los efectos con el gasto público dentro o fuera de la función de utilidad. Los hogares poseen activos que pagan intereses. Estos intereses junto con la renta laboral menos el gasto en consumo, se dedican a la adquisición de nuevos activos, como se refleja en la restricción presupuestaria

$$
\dot{d}(t)=r(t) d(t)+\omega(t) l(t)-c(t)
$$

donde $d(t)$ es la riqueza del hogar, formada por capital y bonos del gobierno; $r(t) \mathrm{y}$ $\omega(t)$ son el tipo de interés y el salario neto de impuestos en términos de consumo en el período $t$.

El agente representativo descuenta el futuro a una tasa $\rho>0$ : Su problema de decisión es:

$$
\begin{aligned}
& \underset{\{c(t), x(t), d(t)\}}{\operatorname{Max}} \quad \int_{0}^{+\infty} U[c(t), x(t), g(t)] e^{-\rho t} d t \\
& \text { sujeto a } \dot{d}(t)=r(t) d(t)+\omega(t) l(t)-c(t) \\
& x(t)+l(t)=1 \text {, } \\
& c(t) \geq 0 \text { para todo } t \text {, } \\
& d(0)=d_{0} \text { dado, }
\end{aligned}
$$

y la condición no Ponzi sobre los activos:

$$
\lim _{t \rightarrow \infty} d(t) \exp \left\{-\int_{0}^{t} r(v) d v\right\} \geq 0 .
$$

Las condiciones de primer orden (CPO) para una solución interior a este problema son:

$$
\begin{gathered}
\theta c(t)^{\theta(1-\sigma)-1} x(t)^{(1-\theta)(1-\sigma)}=\lambda(t), \\
(1-\theta) c(t)^{\theta(1-\sigma)} x(t)^{(1-\theta)(1-\sigma)-1}=\lambda(t) \omega(t),
\end{gathered}
$$

tasa marginal de sustitución entre bienes públicos y privados y el ocio. Para el modelo de aprendizaje por la práctica, si las preferencias sobre el gasto público son separables, como es el caso en este modelo (o si el agente no obtiene ninguna utilidad del gasto público) entonces los efectos riqueza y sustitución se cancelan y el ocio no cambia, lo cual es un requisito para crecimiento equilibrado en este modelo. 


$$
\dot{\lambda}(t)=\lambda(t)[\rho-r(t)],
$$

junto con la condición de transversalidad

$$
\lim _{t \rightarrow 1} e^{-\rho t} \lambda(t) d(t)=0,
$$

donde $\lambda(t)$ es el precio sombra asociado con la restricción presupuestaria del hogar. Las ecuaciones (4)-(5) recogen los dos márgenes básicos del problema. La elección entre $c(0)$ y $c(t)$, ecuación (4), evaluada en 0 y $t$; y la elección entre $c(t)$ y $x(t)$ que iguala la tasa marginal de sustitución al salario real.

\subsection{Empresas}

Hay un gran número de empresas competitivas en la economía que usan como factores productivos capital, mano de obra y gasto público. La empresa representativa produce un bien final según la función de producción Cobb-Douglas con rendimientos constantes a escala dada por

$$
y(t)=A k(t)^{\alpha}\left[l(t) \bar{k}(t)^{\phi} g(t)^{1-\phi}\right]^{1-\alpha},
$$

donde $\alpha \in[0,1], y(t)$ es producto, $A>0$ es un parámetro de escala, $k(t)$ es capital privado, $l(t)$ es mano de obra, $\bar{k}(t)$ denota el nivel de capital agregado, y $g(t)$ es gasto público. El parámetro $\phi=\{0,1\}$ mide el peso relativo de $k(t)$ y $g(t)$ en la función de producción, dadas las dos posibles fuentes de crecimiento endógeno. Bajo estos supuestos los factores reciben como pago sus productos marginales. Esto implica para el capital:

$$
R_{k}(t)=\alpha A k(t)^{\alpha-1}\left[l(t) \bar{k}(t)^{\phi} g(t)^{1-\phi}\right]^{1-\alpha},
$$

y para trabajo:

$$
W(t)=(1-\alpha) A k(t)^{\alpha}\left[\bar{k}(t)^{\phi} g(t)^{1-\phi}\right]^{1-\alpha} l(t)^{-\alpha},
$$

El tipo de interés es igual a la productividad marginal del capital neto de depreciación:

$$
r(t)=R_{k}(t)-\delta,
$$


mientras que el salario neto de impuestos es:

$$
\omega(t)=\left(1-\tau_{w}\right) W(t),
$$

donde $\tau_{w}$ es el impuesto sobre la renta laboral, considerado constante.

\subsection{Gobierno}

En este modelo, el gobierno sigue una senda de gasto público, $g(t)$, que es financiada con impuestos sobre la renta laboral y deuda que adquieren los hogares, es decir, el gobierno no necesita tener un presupuesto equilibrado en cada momento. Se supone que el consumo público es una fracción constante y exógena del total de la producción, $\zeta \in[0,1]$, es decir,

$$
\frac{g(t)}{y(t)}=\zeta,
$$

Con estos supuestos, la restricción presupuestaria del gobierno es:

$$
\dot{b}(t)=R b(t) b(t)+g(t)-\tau_{w} W(t) l(t)
$$

donde $R b(t) b(t)$ denota los gastos en deuda pública y $\tau_{w} W(t) l(t)$ se refiere a los ingresos de impuestos sobre renta laboral. Por último, tenemos la condición no Ponzi sobre la deuda pública:

$$
\lim _{t \rightarrow \infty} b(t) \exp \left\{-\int R b(v) d v\right\} \leq 0 .
$$

Definición 1. En ausencia de límites a la deuda, una política fiscal es un par $\left\{\zeta, \tau_{w}\right\}$ constante a lo largo del tiempo que implica una senda de deuda pública que satisface la condición no Ponzi (15).

\subsubsection{El límite a la deuda}

En este artículo se consideran dos posibles escenarios. En un caso, el gobierno nunca estará restringido a la hora de emitir deuda excepto por la condición no Ponzi (la condición estándar en la literatura); mientras que en el otro caso, se impondrá un 
límite en un momento $T$ sobre el ratio deuda-PIB de la economía. Sea $\chi(t)$ el ratio deuda-PIB, $\frac{b(t)}{y(t)} y(t)$ : Con esta notación, la restricción presupuestaria del gobierno (14) se puede expresar como sigue:

$$
\dot{\chi}(t)=\left[R b(t)-\gamma_{y}(t)\right] \chi(t)+\zeta-\tau_{w}(1-\alpha) .
$$

donde $\gamma_{y}(t)$ es la tasa de crecimiento del producto, $\frac{\dot{y}(t)}{y(y)}$. El segundo caso considerado se recoge en la siguiente gráfica:

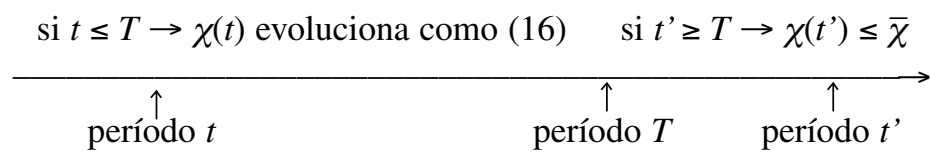

Desde $t \leq T$ la senda para $\chi(t)$ viene dada por la ecuación (16). En un determinado momento, $T$, se impone el límite a la deuda, y el ratio deuda-PIB del gobierno no puede exceder el límite $\bar{\chi}$ : Para simplificar el análisis, se supondrá que una vez que se impone el límite, el gobierno fija el ratio deuda-PIB exactamente en este límite. Por lo tanto, $\chi(t)=\bar{\chi}$ y $\dot{\chi}(t)=0$ : Esto significa que desde $t^{\prime} \geq T$ en adelante, la restricción presupuestaria del gobierno (16) pasa a ser:

$$
\left[r\left(t^{\prime}\right)-\gamma_{y}\left(t^{\prime}\right)\right] \bar{\chi}+\left[1-\tau_{w}(t)\right](1-\alpha)+\zeta-1=0 .
$$

Intuitivamente, restringir la emisión de deuda pública tendrá efectos importantes en el modo de financiar el gasto público. En ausencia de límites, el sector público dispone de dos instrumentos para pagar su gasto, deuda e impuestos. Cuando uno de estos instrumentos se restringe (por ejemplo la deuda), el otro (en este caso los impuestos) tendrá que ajustarse para asegurar que se cumple la restricción presupuestaria (18). Distintos modelos reaccionarán de manera diferente a cambios en los impuestos, y en consecuencia mostrarán distintas sendas de equilibrio. Por lo tanto, la política fiscal en cada escenario será distinta.

Definición 2. Si hay un límite a la deuda, una política fiscal es un par $\left\{\zeta, \tau_{w}\right\}$ constante a lo largo del tiempo, y donde la deuda pública viene determinada por la ecuación (14). Cuando se impone el límite, la política fiscal es una constante $\zeta, y$ una senda de $\tau_{w}(t)$ que satisfacen la ecuación (18). 


\section{Equilibrio competitivo}

Dada una política fiscal, se combinan las condiciones de maximización de la utilidad con las de maximización de beneficios, junto con la restricción presupuestaria del gobierno y las condiciones de vaciado de mercados para caracterizar el equilibrio competitivo de esta economía. La especificación de Cobb-Douglas para la función de utilidad junto con los rendimientos a escala constantes de la función de producción permiten la existencia de crecimiento endógeno ${ }^{3}$.

Nótese que cuando $\eta=0$, y $\phi=0$, el modelo se convierte en un escenario $\grave{a}-l a$ Barro, en el cual el gasto del gobierno entra en la función de producción potenciando tanto la productividad del capital como la del trabajo. Sin embargo, si $\eta=1$, y $\phi=1$, el gasto público entra aditivamente en la función de utilidad, y la función de producción exhibe externalidades debido al conocimiento y el aprendizaje por la práctica à-la-Romer. En adelante, me referiré al primer caso $(\eta=0$, y $\phi=0)$ como el modelo con Gasto público en la Función de Producción (modelo GFP), y al segundo caso ( $\eta=1$, y $\phi=1)$ como el modelo con Gasto público en la Función de Utilidad (modelo GFU). En equilibrio, suponiendo simetría entre las empresas, los stocks de capital agregado e individual son iguales, $\bar{k}(t)=k(t)$. Usando la ecuación (13), el nivel de producción pasa a ser:

$$
y(t)=\left[A k(t)^{\alpha+\phi(1-\alpha)} l(t)^{1-\alpha} \xi^{(1-\alpha)(1-\phi)}\right]^{\varphi} .
$$

donde $\varphi=\frac{1}{1-(1-\phi)(1-\alpha)}$.

Definición 3 Tomando como dados el estado inicial, $k(0)$ y $b(0)$, y una política fiscal, una senda de equilibrio competitivo para la economía descrita anteriormente consiste en unas secuencias para las cantidades $\{c(t), l(t), k(t), b(t)\}_{t=0}^{\infty}, y$ precios $\{r(t), \omega(t)\}_{t=0}^{\infty}$, tales que:

(i) la tripleta $\{c(t), x(t), \dot{k}(t)\}_{t=0}^{\infty}$ resuelve el problema del hogar representativo,

(ii) el par $\{l(t), k(t)\}_{t=0}^{\infty}$ resuelve el problema de la empresa representativa,

(iii) el mercado de trabajo se vacía,

\footnotetext{
${ }^{3}$ Para una discusión más detallada a este respecto, véase King, Plosser y Rebelo (1988).
} 


$$
x(t)+l(t)=1
$$

el mercado de bienes se vacía,

$$
\dot{k}(t)=y(t)-c(t)-g(t)-\delta k(t)
$$

y los mercados de capitales se vacían,

$$
d(t)=k(t)+b(t)
$$

(iv) se cumple la restricción presupuestaria del gobierno (14),

$$
\dot{b}(t)=R_{b}(t) b(t)+g(t)-\tau_{w}(t) W(t) l(t)
$$

(v) y debido al no arbitraje, el capital y la deuda pública pagan el mismo tipo de interés,

$$
r(t)=R_{b}(t)=R k(t)-\delta .
$$

Las condiciones de primer orden que caracterizan el equilibrio competitivo se muestran en el Apéndice.

\section{Senda de CRECIMiento Equilibrado}

En esta sección, el análisis se centra en la senda de crecimiento equilibrado, con el fin de estudiar los efectos a largo plazo de la política fiscal ${ }^{4}$.

Definición 4. Una senda de crecimiento equilibrado es aquella en la que el consumo, el gasto público, el producto, la deuda y el capital crecen a la misma tasa, $\gamma$, y en la que la asignación del tiempo (trabajo, ocio), el tipo de interés, los salarios y las variables fiscales, $\tau_{w} y \zeta$, son constantes a lo largo del tiempo.

${ }^{4}$ Para denotar las variables en el estado estacionario, se elimina la referencia al tiempo entre paréntesis. 
En la senda de crecimiento equilibrado, todas las variables crecen a la misma tasa $\gamma$

$$
\gamma=\frac{1}{1-\theta(1-\sigma)}\left(R_{k}-\delta-\rho\right)
$$

donde es necesario que $R_{k}>\rho+\delta>\theta(1-\sigma) \gamma+\delta$, para asegurar tanto el crecimiento endógeno como la utilidad acotada, respectivamente. Las variables que crecen están expresadas como ratios del capital, $k(t)$.

En esta economía, la senda de crecimiento equilibrado (en adelante, SCE) viene descrita por el conjunto de variables $\left\{\gamma, l, \frac{c}{k}, \frac{y}{k}, \frac{b}{k}, \tau_{w}\right\}$ en ausencia de límite. Si hay un límite a la deuda, la SCE viene descrita bien por $\left\{\gamma, l, \frac{c}{k}, \frac{y}{k}, \tau_{w}\right\}$ o por $\left\{\gamma, l, \frac{c}{k}, \frac{y}{k}, \zeta\right\}$, dependiendo de qué variable se ajusta para satisfacer el límite. Estas variables resuelven el siguiente sistema de ecuaciones:

$$
\begin{gathered}
\theta(1-l)\left(1-\tau_{w}\right)(1-\alpha) \frac{y}{k l}=(1-\theta) \frac{c}{k}, \\
\gamma=\frac{1}{1-\theta(1-\sigma)}\left[\alpha \frac{y}{k}-\delta-\rho\right], \\
\frac{y}{k}=A\left[l\left(\zeta \frac{y}{k}\right)^{(1-\phi)}\right]^{1-\alpha} \\
\gamma=\frac{y}{k}-\zeta \frac{y}{k}-\frac{c}{k}-\delta
\end{gathered}
$$

y si no hay límite a la deuda

$$
\xi \frac{y}{k}+\left(\alpha \frac{y}{k}-\delta-\gamma\right) \frac{b}{k}-\tau_{w}(1-\alpha) \frac{y}{k}=0
$$

o si hay un límite $\bar{\chi}$, 


$$
\zeta+\left(\alpha \frac{y}{k}-\delta-\gamma\right) \bar{\chi}-\tau_{w}(1-\alpha)=0
$$

La ecuación (19) representa la decisión de ofertar trabajo por los hogares y depende del salario neto de impuestos y del consumo. La ecuación (20) es la tasa de crecimiento del consumo que resulta del problema de optimización del individuo. La ecuación (21) es la función de producción en términos del ratio producto-capital y del trabajo. La ecuación (22) es la restricción de consistencia agregada. Finalmente, las dos ecuaciones siguientes, (23) y (24), representan la restricción presupuestaria del gobierno sin y con límites, respectivamente.

En presencia de límite a la deuda, $\bar{\chi}$, el ratio $\frac{b}{k}$ viene determinado por $\frac{y}{k}$, ya que imponer un límite implica que $\frac{b}{k}=\bar{\chi} \frac{y}{k}$, donde $\bar{\chi}$ está fijo. Esto significa que cualquier cambio en la política fiscal a través de impuestos, $\tau_{w}$, hace $\zeta$ endógeno, mientras que cambios en el ratio gasto público-PIB, $\zeta$, hará que los impuestos sean endógenos.

\section{PARÁMetros}

En general, no es posible resolver analíticamente estos modelos. De hecho, se puede obtener una solución cerrada en ciertas versiones del modelo, pero no cuando se hace endógena la decisión trabajo-ocio, como es el caso aquí. Para entender las consecuencias de imponer límites a la deuda pública y compararlas con el caso estándar, se simula el modelo utilizando valores de los parámetros que son estándar en la literatura.

Los parámetros del modelo son $\sigma, \theta, \psi, \alpha, \delta, \tau_{w}, \rho, A$ y $\zeta$. La Tabla 1 recoge los valores de los parámetros utilizados y la Tabla 2 muestra los valores para las principales variables en el estado estacionario.

La elasticidad de sustitución intertemporal, $\sigma$, es igual a 2: La elasticidad de sustitución entre consumo y ocio, $\theta$, está calibrada para reproducir un porcentaje de ocio sobre horas trabajadas de alrededor del 0.4, como sugieren los datos para Estados Unidos. El factor de descuento, $\rho$, está calibrado para obtener un tipo de interés anual del 4\%: La elasticidad de sustitución entre bienes privados y públicos en la función de utilidad, $\psi$, no tiene efecto sobre la senda de crecimiento equilibrado ya que $g(t)$ no es una variable de elección para el hogar. Por ello, no es necesario asignarle ningún valor. 
Tabla 1. Valores de los parámetros

\begin{tabular}{|l|c|}
\hline Parámetro de tecnología medolo GFU & $\phi=1, \quad \mathrm{~A}=0.1799$ \\
Parámetro de tecnología medolo GFP & $\phi=1, \quad \mathrm{~A}=2.1494$ \\
Participación del capital sobre el producto & $\alpha=1 / 3$ \\
Tasa de depreciación & $\delta=0.0238$ \\
Ratio gasto público-PIB & $\zeta=0.24$ \\
Impuesto sobre la renta laboral & $\tau_{w}=0.3647$ \\
Inversa de la elasticidad de sustitución intertemporal & $\sigma=2$ \\
Factor de descuento & $\rho=0.0026$ \\
Elasticidad de sustitución entre consumo y ocio & $\theta=0.4481$ \\
\hline
\end{tabular}

Tabla 2. Valores de la senda de crecimiento equilibrado*

\begin{tabular}{|l|c|}
\hline Tasa de crecimiento $(\gamma)$ & 0.0050 \\
Tipo de interés nominal $(r)$ & 0.0098 \\
Ratio consumo-capital $(c / k)$ & 0.0479 \\
Ratio gasto público-capital $(g / k)$ & 0.0242 \\
Ratio producto-capital $(y / k)$ & 0.1009 \\
Ratio deuda pública-capital $(b / k)$ & 0.0656 \\
Empleo $(l)$ & 0.4198 \\
\hline
\end{tabular}

* Para facilitar la comparación, los valores de estado estacionario son los mismos para los dos modelos (GFU y GFP).

Como en Stokey y Rebelo (1995), se comparan dos economías que son observacionalmente equivalentes: parten alrededor de la misma senda de crecimiento equilibrado, pero responden de modo distinto a cualquier cambio en los parámetros. Para partir de los dos modelos en el mismo estado estacionario, el ajuste se hace vía el parámetro de tecnología, $A$ : La tasa de depreciación anual, $\delta$, es igual a $10 \%$. La participación del capital en el producto, $\alpha$, es $1=3$.

Respecto a las variables fiscales, es necesario determinar el tipo impositivo sobre la renta, $\tau_{w}$, y el peso del gasto público sobre el PIB, $\zeta$. El impuesto sobre la renta laboral se ha fijado en $\tau_{w}=36.47 \%$, lo que corresponde a un ratio gasto público-PIB, $\zeta$, del $24 \%$. Todos estos valores implican un ratio deuda-PIB, $\chi$, igual a $65 \%$. 


\section{EFectos A LARGo PLAZO DE LA POLÍticA FISCAL}

En principio, para gestionar su presupuesto, el gobierno tiene tres instrumentos, deuda, impuestos y gasto público. El restringir uno de ellos (en este caso la deuda) afecta la asignación de los otros (impuestos y gasto público). Para controlar la deuda pública (bien para reducir la cantidad de deuda emitida o simplemente para prevenir que crezca sin control) el gobierno puede aumentar los impuestos o reducir el gasto público.

En esta sección, se analizan los efectos a largo plazo de dos políticas fiscales (cambios en los impuestos sobre la renta, $\tau_{w}$, y en el ratio gasto público-PIB, $\zeta$ ) en los dos modelos considerados (GFP y GFU), destacando las diferencias introducidas por la existencia de límites a la deuda. Este análisis se hará sin tener en cuenta la dinámica transicional.

\subsection{Un aumento del impuesto sobre la renta $\left(\tau_{w}\right)$}

El primer experimento consiste en aumentar el impuesto sobre la renta del $36.47 \%$ al $41.47 \%$, manteniendo todo los demás parámetros constantes. Las Figuras 1 y 2 muestran los resultados para los modelos GFP y $G F U$, respectivamente. Las líneas continuas se refieren a la economía sin límites a la deuda, y las discontinuas a la economía con límites a la deuda. La Figura 3 muestra los efectos sobre la tasa de crecimiento del producto y el ratio deuda-PIB bajo límites para los dos modelos considerados, el GFP (línea continua) y el GFU (línea discontinua).

Como era de esperar, los efectos a largo plazo de un aumento de los impuestos difieren según el papel representado por el gasto público en el modelo. En ausencia de límites a la deuda en el modelo GFP, un aumento del impuesto sobre la renta tiene dos efectos opuestos sobre la oferta de trabajo. Por un lado, disminuye los salarios realmente obtenidos por los hogares. Esto reduce sus incentivos a trabajar, afectando negativamente al producto, la recaudación impositiva y por lo tanto al crecimiento. Por otro lado, tiene un efecto positivo sobre el gasto público, y afecta positivamente la productividad del trabajo, aumentando la oferta de trabajo. En las figuras, el primer efecto domina, con lo que el trabajo, la producción y el consumo privado caen y por lo tanto la tasa de crecimiento de la economía. Dado que el gasto del gobierno es una proporción constante $\zeta$ del producto, el consumo público también disminuye, acentuando la caída de la tasa de crecimiento. 
Figura 1. Cambios en el modelo GFP para distintos impuestos sobre la renta. La línea continua se refiere al modelo sin límites de deuda, y la discontinua al modelo con límites
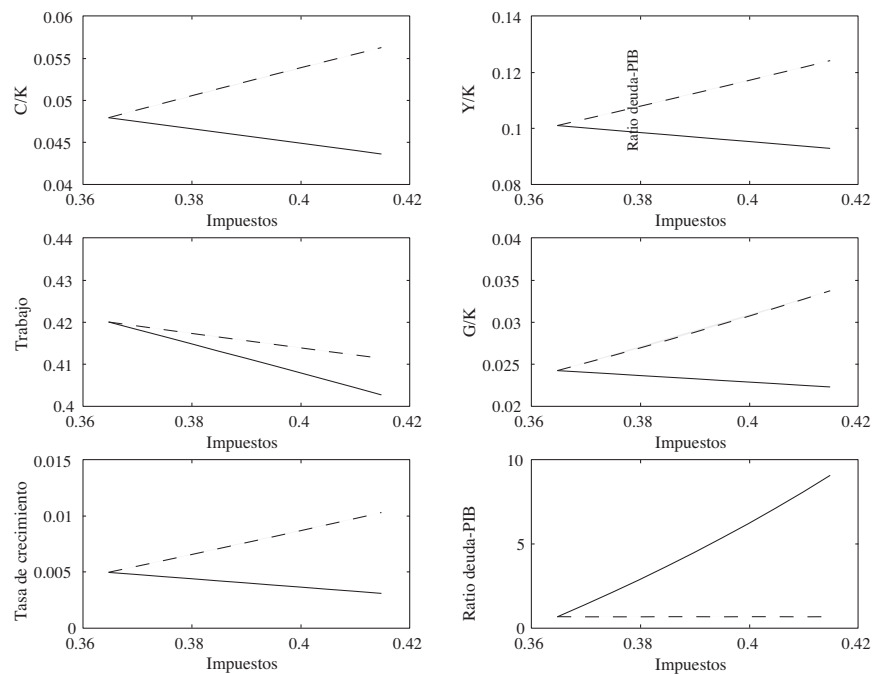

Figura 2. Cambios en el modelo GFU para distintos impuestos sobre la renta. La línea continua se refiere al modelo sin límites de deuda, y la discontinua al modelo con límites
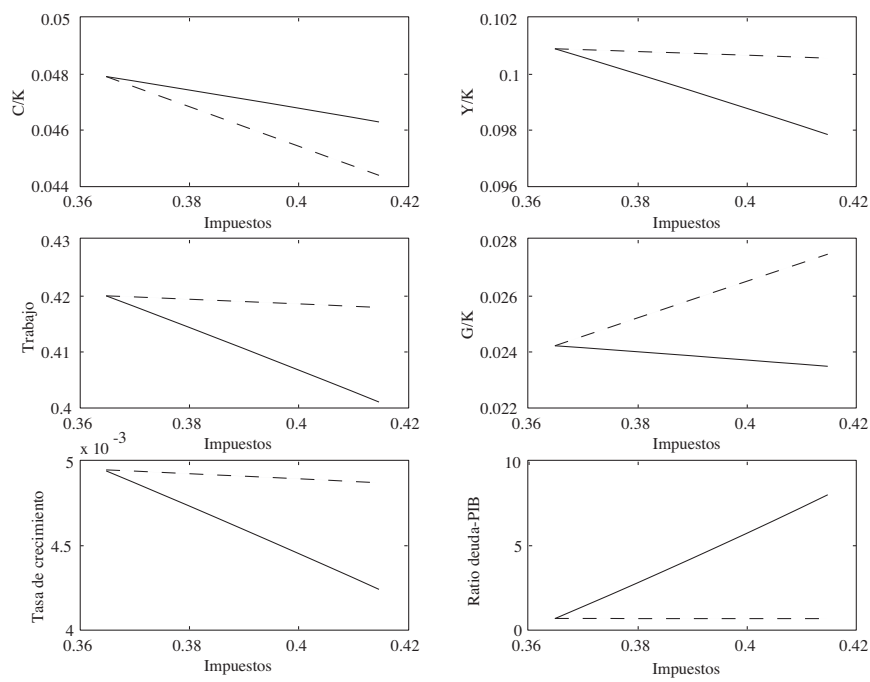
Figura 3. Cambios en los modelos GFP y GFU para distintos impuestos sobre la renta. La línea continua se refiere al modelo GPF y la discontinua al modelo GFU

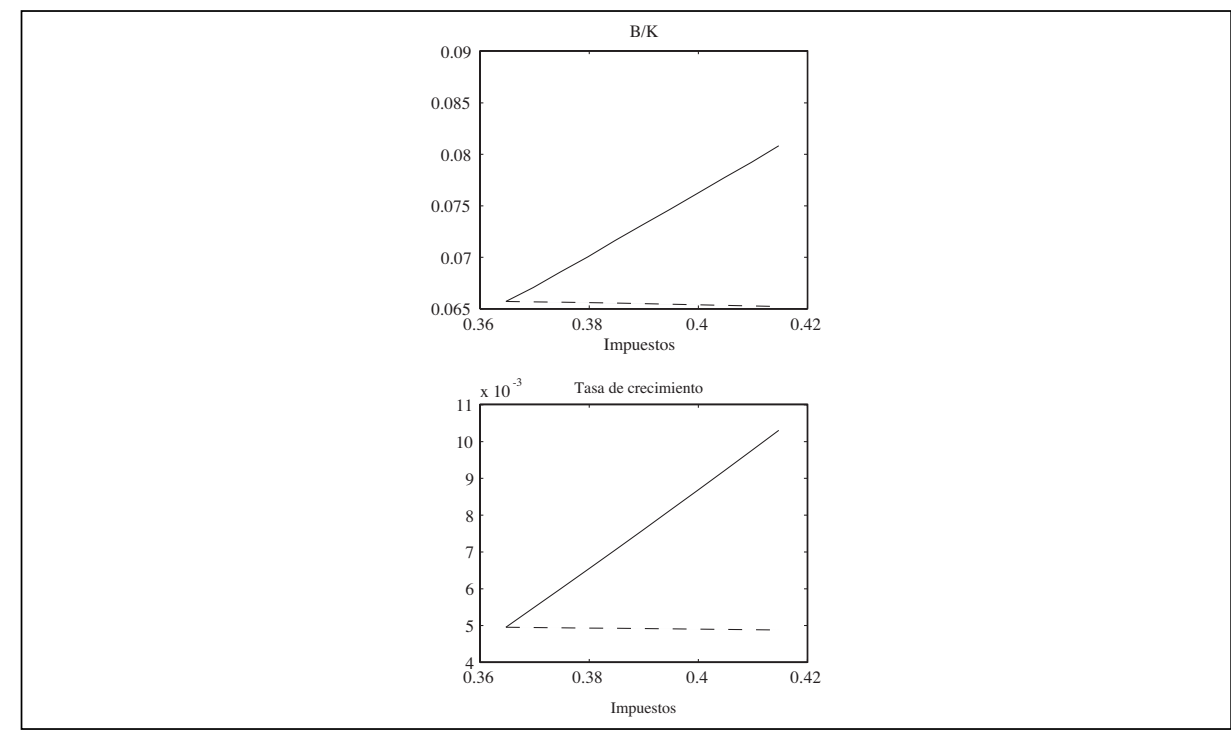

Con límites a la deuda, los dos efectos contrarios del aumento de impuestos sobre la renta en la oferta de trabajo aún están presentes. Sin embargo, como el ratio deuda pública-PIB no puede cambiar, la mayor recaudación impositiva se dedica completamente a aumentar el gasto público. En el modelo GFP, más gasto público aumenta la tasa de crecimiento de la economía. Por lo tanto, a diferencia del modelo sin límites, el resultado final es un aumento de la producción, gasto público y crecimiento.

Los mismos resultados aparecen para el modelo GFU en ausencia de límites. Nótese que los efectos sobre la tasa de crecimiento son mayores en el modelo GFP que en el GFU. La razón es que en el modelo GFU, más gasto público no afecta a la productividad del trabajo, mientras que los impuestos sobre la renta sí, reduciendo los incentivos a trabajar, con un efecto negativo sobre el crecimiento. Como resultado, un aumento de los impuestos reduce tanto el consumo público como el privado. Cuando hay un límite a la deuda pública, el aumento de los impuestos permite un mayor gasto público, atenuando los efectos negativos de la política fiscal. 


\subsection{Una reducción del ratio gasto público-PIB (ङ)}

A continuación, se consideran los efectos a largo plazo de una reducción del tamaño del sector público, $\zeta$, del $24 \%$ al $22 \%$. La Figura 4 hace referencia al modelo GFP, y la Figura 5 muestra el modelo GFU. Como antes, las líneas continuas se refieren a la economía sin límites de deuda, y las discontinuas a la economía con un límite a la deuda. La Figura 6 compara ambos modelos cuando se impone el límite.

La Figura 4 muestra que en el modelo GFP, reducir $\zeta$ afecta negativamente a todas las variables. Estos efectos son menos pronunciados (o incluso positivos como en el caso del trabajo) si hay un límite a la deuda. En este caso, un cambio en ? conlleva un cambio en los impuestos para mantener equilibrada la restricción presupuestaria del gobierno (24). Con la deuda controlada por el límite, el impuesto inducido por un menor $\zeta$ no tiene que ser tan alto como antes. Esto tiene un efecto positivo sobre la mano de obra, evitando que caiga.

Sin embargo, en el modelo GFU la misma caída en $\zeta$ sólo afecta al bienestar de los individuos, sin efecto directo sobre el crecimiento. La Figura 5 muestra que un

Figura 4. Cambios en el modelo GFP para distintos $\zeta$. La línea continua se refiere al modelo sin límites de deuda, y la discontinua al modelo con límites

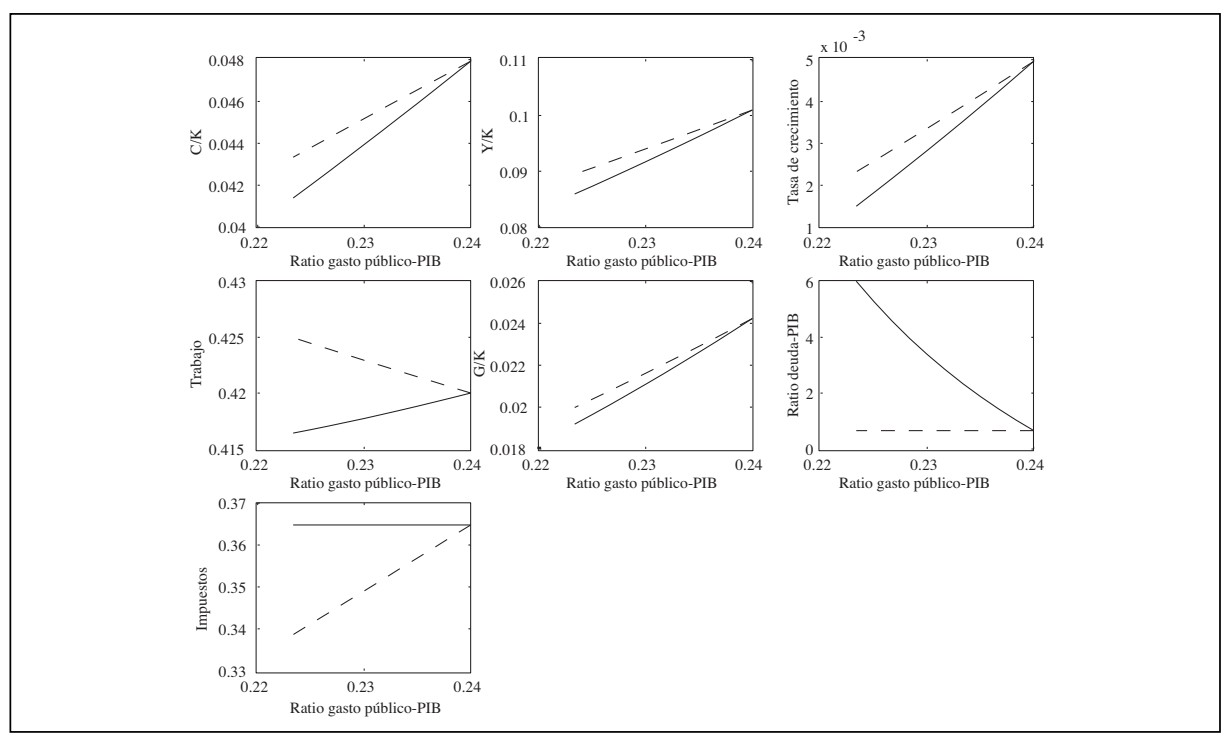


Figura 5. Cambios en el modelo GFU para distintos $\zeta$. La línea continua se refiere al modelo sin límites de deuda, y la discontinua al modelo con límites

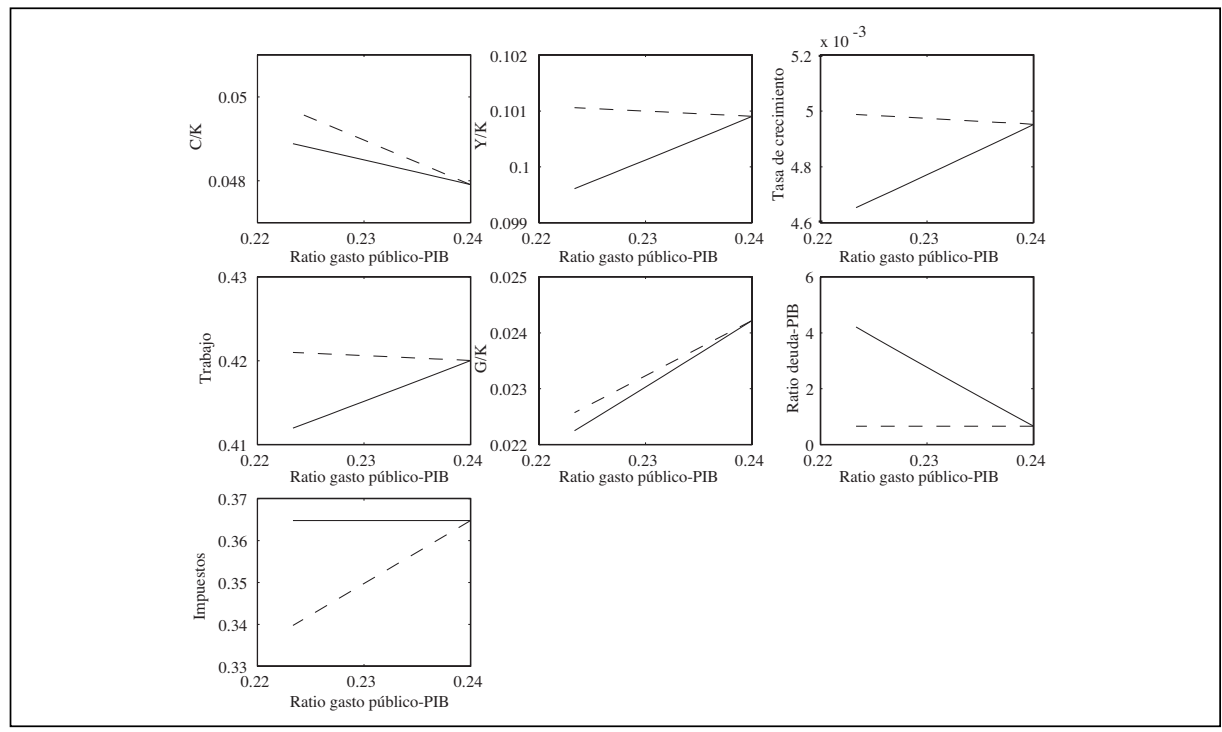

Figura 6. Cambios en los modelos GFP y GFU para distintos $\zeta$. La línea continua se refiere al modelo GFP y la discontinua al modelo GFU

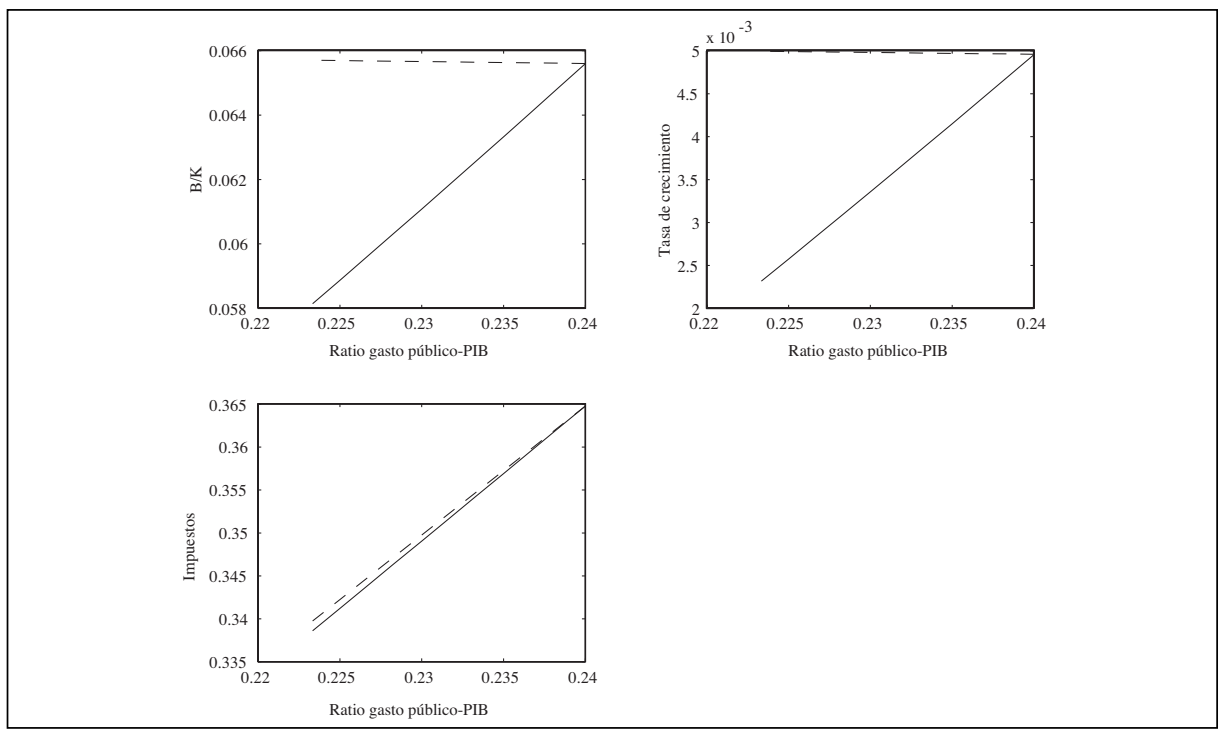


menor tamaño del sector público reduce el producto, y el trabajo. El efecto final sobre el crecimiento es negativo. Al mantener los impuestos constantes, los recursos de reducir $\zeta$ se dirigen a aumentar la emisión de deuda. Sin embargo, en la economía con el límite de deuda, la caída en $\zeta$ tiene el efecto contrario a un aumento de los impuestos, es decir, aumenta la tasa de crecimiento.

\section{Dinámica transicional}

Aunque el análisis anterior se centraba en la senda de crecimiento equilibrado, los dos modelos considerados en este artículo tienen dinámicas transicionales. En esta sección se analiza la dinámica transicional en el modelo GFP cuando la política fiscal está restringida con el fin de reducir el ratio deuda-PIB hasta el nuevo límite. La política fiscal en este análisis tomará dos formas distintas. En ausencia de límites, la política fiscal se definía como el par $\left\{\zeta, \tau_{w}\right\}$ constante a lo largo del tiempo y que implicaba una senda de deuda pública consistente con la condición no Ponzi (15). En presencia de límites a la deuda, la política fiscal consistirá en una constante $\zeta$ y una senda para $\tau_{w}(t)$ que mantengan el ratio deuda-PIB constante e igual al límite impuesto, $\bar{\chi}$ : Para simplificar, consideraré el caso en que sólo hay un período de transición entre regímenes, esto es, $T=1$ en el esquema $(17)^{5}$.

\subsection{Un aumento del impuesto sobre la renta $\left(\tau_{w}\right)$}

En esta sección, se analiza la dinámica transicional de una economía que aumenta sus impuestos con el fin de reducir el ratio deuda-PIB. Como he mencionado antes, el momento en que el límite es aplicado es $T=1$ : El objetivo es comparar las dinámicas fuera de la SCE tras el aumento de los impuestos con la SCE inicial, el escenario de referencia.

La Figura 7 muestra los resultados de un aumento temporal del impuesto sobre la

${ }^{5}$ Este es el modo más sencillo de estudiar los efectos dinámicos de imponer el límite, ya que se evita calcular todas las posibles dinámicas entre el momento del anuncio y el momento en que el límite se hace activo, $T$. Sin embargo, períodos de transición más largos se dejan para futuros trabajos. 
renta laboral del $36.47 \%$ al $40 \%$, lo que implica una reducción del ratio deuda-PIB del $65 \%$ al $60 \%$. En las figuras, la línea continua se refiere al modelo sin límites a la deuda, y la discontinua al modelo con límites.

Tras el cambio inicial en impuestos, la deuda se reduce para alcanzar el límite impuesto. Dado que $\frac{b}{y} y$ está restringido por el límite, $\tau_{w}$ pasa a ser endógeno, y convergen a una nueva SCE con menores impuestos sobre la renta. Esto afecta positivamente al trabajo. Aunque parezca contraintuitivo, menores impuestos conllevan un mayor gasto público. Dado el supuesto de un ratio de gasto fijo, $\zeta=\frac{g}{y}$, y dado el papel productivo del gasto público en esta economía, menores distorsiones vía impuestos resultan en mayor producto y mayor gasto público al tiempo que aumentan el consumo y el crecimiento.

Esto es, si la economía aumenta sus impuestos sobre la renta para reducir la deuda y mantenerla a un ratio fijo sobre el PIB, la economía convergerá a una nueva SCE en la que el consumo, producto, trabajo y crecimiento serán mayores, los impuestos menores y el gasto público aumentará respecto a una economía que permanece en su SCE inicial.

Figura 7. El modelo GFP tras un aumento del impuesto sobre la renta. La línea continua srefiere al modelo sin límites a la deuda, y la discontinua al modelo con límites. Todas las variables están expresadas como porcentajes respecto a sus valores de SCE iniciales

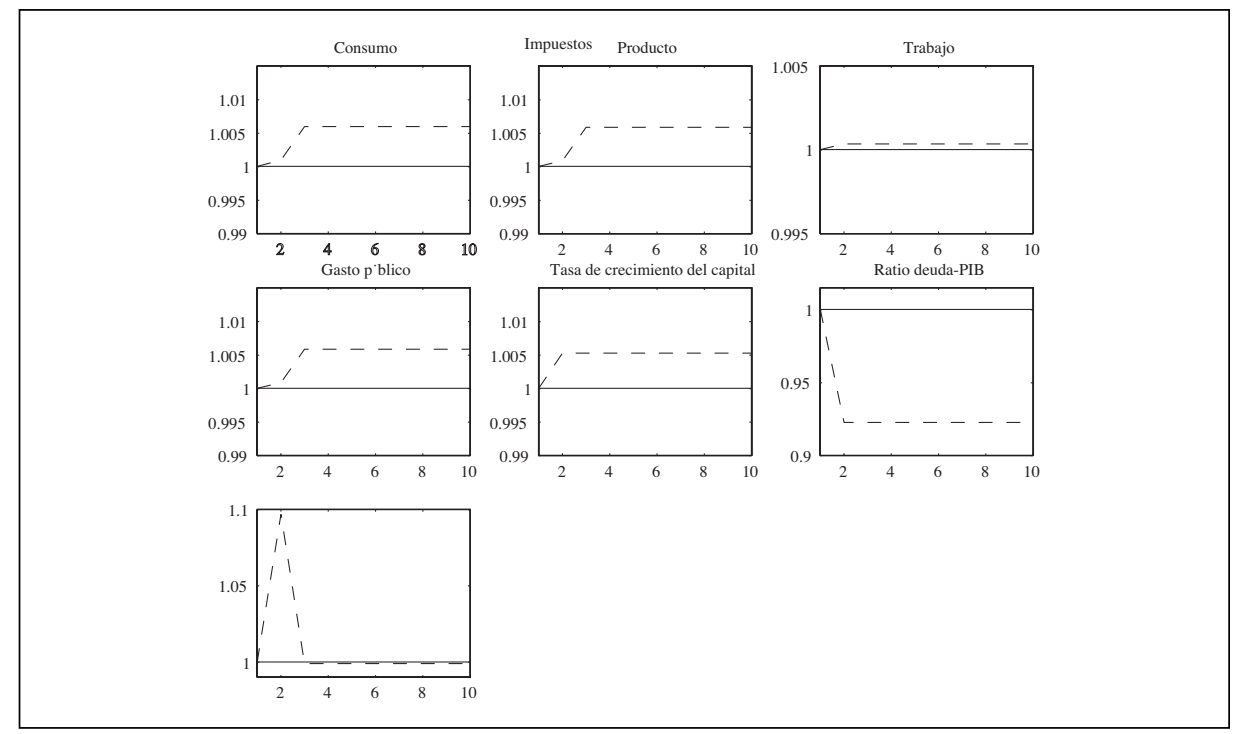




\subsection{Una reducción del ratio gasto público-PIB (६)}

Siguiendo un análisis paralelo a la sección anterior, aquí se analiza una reducción del ratio gasto público-PIB, $\zeta$, del $24 \%$ al $22 \%$, de una vez y para siempre en $T=1$ : En este caso, la economía utiliza los cambios en $\zeta$ para reducir su ratio deuda-PIB y alcanzar otra SCE con el límite. Como antes, se comparan dos casos, sin límites (escenario de referencia), y con límites. Como en el caso anterior, la línea continua se refiere al modelo sin límites a la deuda, y la discontinua al modelo con límites. La Figura 8 recoge los resultados.

Reducir el gasto público para disminuir el ratio deuda-PIB, afecta negativamente al consumo, producción y tasa de crecimiento. Un menor consumo público y deuda permiten a la economía disfrutar de menores impuestos sobre la renta. El efecto inmediato es un aumento de la oferta de trabajo. Por ello, la reducción en $\zeta$ para alcanzar un menor nivel de deuda-PIB por debajo del inicial, lleva a la economía a una nueva SCE con menor consumo, producción, tasa de crecimiento, gasto público, e impuestos y un mayor empleo.

La principal diferencia entre esta política fiscal y la anterior está en el signo de los efectos. Aunque ambas políticas conducen a una reducción de la cantidad de deuda, las dinámicas fuera de los estados estacionarios confirman los resultados obtenidos previamente en el análisis de largo plazo: aumentar los impuestos en presencia de límites a la deuda afecta positivamente la tasa de crecimiento. Ahora, la dinámica añade qué ocurre con el consumo y empleo. Con impuestos inicialmente más altos sobre la renta, el hogar representativo disfruta de un mayor consumo y menores impuestos en el futuro. Cuando se reduce el gasto público, el consumo y empleo son menores.

\section{ANÁlisis DE BIENESTAR}

En esta sección, se estudian los efectos sobre el bienestar de los cambios en la política fiscal analizados anteriormente en la economía con límites a la deuda y para el modelo GFP. El coste en bienestar de implementar una determinada política fiscal se obtiene de comparar los niveles de bienestar en la SCE inicial y durante la transición entre SCEs. El bienestar sobre la SCE, $W_{S C E}$, viene dado por 


$$
W_{S C E}=\int_{0}^{+\infty} U\left[c_{S C E} x_{S C E}\right] e^{-\rho t} d t=\int_{0}^{+\infty}\left[\frac{\left(c^{\theta}{ }_{S C E}^{1-\theta} x_{S C E}\right)^{1-\sigma}}{1-\sigma}\right] e^{-\rho t} d t=\int_{0}^{+\infty}\left[\frac{\left(c_{0}^{\theta} e^{\theta \gamma_{0} t} x^{1-\theta}\right)^{1-\sigma}}{1-\sigma}\right] e^{-\rho t} d t
$$

donde $c_{0}$ y $x_{0}$ denotan la SCE inicial, y donde todas las variables crecen a la misma tasa, $\gamma_{0}$.

El nivel de bienestar alcanzado durante la dinámica transicional, $W_{T D}$, viene dado por

$$
W_{T D}=\int_{0}^{+\infty} U\left[c_{T D}, x_{T D}\right] e^{-\rho t} d t=\int_{0}^{+\infty}\left\{\frac{\left[c(t)^{\theta} x(t)^{1-\theta}\right]^{1-\sigma}}{1-\sigma}\right\} e^{-\rho t} d t
$$

El coste en términos de bienestar de políticas fiscales se mide como el porcentaje de consumo en la SCE inicial que el agente estaría dispuesto a perder con el fin de no experimentar ningún cambio en el consumo tras el cambio en política fiscal (Lucas, 1987). Este coste es $\varsigma$, y se puede calcular como sigue:

$$
W_{S C E}=\int_{0}^{+\infty} U\left[c_{S C E}(1-\varsigma), x_{S C E}\right] e^{-\rho t} d t=W_{T D}
$$

esto es,

$$
\varsigma=1-\left[\frac{W_{T D}}{W_{S C E}}\right]^{\frac{1}{\theta(1-\sigma)}}
$$

La Tabla 3 muestra el coste en términos de bienestar, $\varsigma$, de las dos políticas fiscales analizadas en presencia de límites a la deuda. El coste asociado con un aumento de los impuestos es menor que cuando se reduce el ratio gasto público-PIB. En el primer caso, se debe al aumento del empleo y de la tasa de crecimiento, que llevan a la economía a una nueva SCE con mayores niveles de consumo. Cuando se reduce el gasto público, el coste en bienestar es mucho mayor, debido a la reducción del consumo y al aumento del empleo como se observa en la Figura 8. 
Tabla 3. Efectos sobre el bienestar de las políticas fiscales

\begin{tabular}{|lc|}
\hline & Coste en términos de bienestar $(\varsigma)$ \\
\hline Un aumento del impuesto sobre la renta con límites & $14.62 \%$ \\
\hline Una reducción del ratio gasto público-PIB con límites & $25.71 \%$ \\
\hline
\end{tabular}

El coste en términos de bienestar de las políticas fiscales, $\varsigma$, está expresado en porcentaje del consumo en la SCE inicial.

Figura 8. El modelo GFP tras una reducción en $\zeta$. La línea continua se refiere al modelo sin límites de deuda, y la discontinua al modelo con límites. Todas las variables están expresadas como porcentajes respecto a sus valores de SCE iniciales
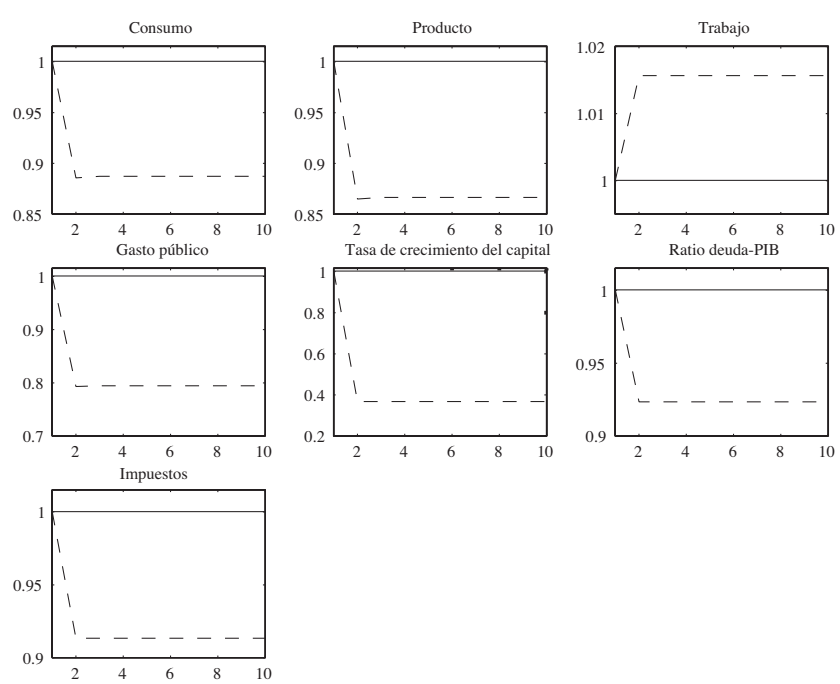

\section{Conclusiones}

El objetivo de este artículo es investigar las consecuencias sobre el crecimiento y el bienestar de imponer límites a la deuda en la restricción presupuestaria del gobierno. Para ello se analizan dos modelos que difieren en el papel asignado al gasto público, bien como gasto productivo (en la función de producción), o suministrando servicios públicos (en la función de utilidad), en este último caso, siendo el capital privado el motor del crecimiento. 
En el largo plazo, si no hay límites a la deuda pública, aumentar los impuestos sobre la renta reduce el crecimiento, independientemente del papel asignado al gasto público, y viceversa. En presencia de un límite sobre la deuda pública, mayores impuestos sobre la renta tienen un efecto positivo sobre el crecimiento siempre que el gasto público es productivo.

También se analiza la dinámica transicional hacia una nueva senda de crecimiento equilibrado con menor ratio deuda-PIB en el modelo con gasto productivo. Los resultados indican que aumentar los impuestos para reducir la deuda pública lleva a la economía a una nueva SCE con mayor crecimiento y menores impuestos. Del mismo modo, reducir el tamaño del sector público tiene los efectos contrarios, menor crecimiento y producción, debido al papel del gasto público. Finalmente, en presencia de límites a la deuda, mayores impuestos sobre la renta implican un menor coste en términos de bienestar que reducir el gasto público. La razón está en los mayores niveles de consumo que el hogar representativo disfruta si los impuestos son el instrumento de política fiscal. 
ApÉNDICE I. CONDICIONES DE PRIMER ORDEN DEL EQUiLIBRIO COMPETITIVO

Las condiciones de equilibrio competitivo en el modelo general son

$$
\begin{gathered}
\frac{\theta\left(1-\tau_{w}\right) W(t)}{(1-\theta)}=\frac{c(t)}{[1-l(t)]}, \\
\frac{\dot{\lambda}(t)}{\lambda(t)}=[\rho-r(t)], \\
\frac{\dot{c}(t)}{c(t)}=\frac{1}{\theta(1-\sigma)-1}\left[(1-\theta)(1-\sigma) \frac{\dot{l}(t)}{1-l(t)}+\frac{\dot{\lambda}(t)}{\lambda(t)}\right], \\
r(t)=\alpha \frac{y(t)}{k(t)}-\delta, \\
W(t)=(1-\alpha) \frac{y(t)}{l(t)}, \\
\dot{k}(t)=y(t)-g(t)-c(t)-\delta k(t), \\
\dot{b}(t)=r(t) b(t)-g(t)-\tau_{w} W(t) l(t), \\
g(t)=\xi y(t), \\
A k(t)^{\alpha}[l(t) g(t)]^{1-\alpha} \\
A k(t) l(t)^{1-\alpha}, \\
\lim _{t \rightarrow 0} e^{-\rho t} l(t) d(t)=0,
\end{gathered}
$$

donde $\lambda(t)$ es el precio sombra asociado a la restricción presupuestaria del hogar. Las ecuaciones (A1), (A2) y (A3) describen las elecciones óptimas del hogar. Las condiciones (A4), y (A5) son las demandas de factores por parte de las empresas. Las ecua- 
ciones (A6) y (A7) muestran las leyes de movimiento de las dos variables estado del sistema. La ecuación (A8) describe la política fiscal, la ecuación (A9) especifica la función de producción dependiendo del modelo considerado, y la ecuación (A10) es la condición de transversalidad. Por último, está la restricción $l(t) \in[0,1]$.

\section{ApÉNDICE II. OBtención DE LA DINÁMICA TRANSICIONAL}

Para recuperar la senda de equilibrio de las variables se utiliza el siguiente procedimiento.

1. El conjunto de condiciones de optimalidad para el equilibrio competitivo (ecuaciones A1-A10 en el Apéndice) tiene que expresarse en términos de las variables normalizadas. Por ello, todas las variables que crecen se expresan en ratios respecto del capital, $k(t)$.

2. El sistema se reduce al menor número de variables posible. El vector de incógnitas es $z(t)=\{\beta(t), \eta(t), \xi(t), l(t)\}$, donde $\beta(t)=\frac{b(t)}{k(t)}, \eta(t)=\frac{c(t)}{k(t)}, \mathrm{y} \xi(t)=\frac{y(t)}{k(t)}$.

Cuando se impone el límite a la deuda, hay una ecuación adicional (la que surge del límite de deuda), y una incógnita más $\tau_{w}(t)$. Por ello, $z(t)$ pasa a ser $z^{\prime}(t)=\left\{\beta(t), \eta(t), \xi(t), l(t), \tau_{w}(t)\right\}$.

3. Para recuperar la senda de las series originales, es necesario caracterizar la senda de crecimiento equilibrado a la que convergerían las nuevas variables. Dada la no-linealidad del modelo resultante, se linearías el modelo alrededor de la nueva senda de crecimiento equilibrado para resolverlo. El sistema linearizado tiene la siguiente estructura:

$$
A \tilde{z}(t)+B \tilde{z}(t)=0 \text {, esto es, } \tilde{z}(t)=P \tilde{z}(t)
$$

con $P=-A^{-1} B$, y $\tilde{z}(t)=z(t)-\tilde{z}$, donde $\tilde{z}$ denota las variables en la nueva senda de crecimiento equilibrado. Una vez que el sistema está resuelto, se obtiene la senda del vector $\tilde{z}(t)$ en términos de $\Lambda$, la matriz de valores propios estables de la matriz $P$. Las condiciones de estabilidad requieren que las series resultantes no sean explosivas, es decir, en tiempo continuo que los elementos de $\Lambda$ sean negativos. 


\section{BiBLIOGRAFÍA}

Baier, S. L. y G. Glomm, 2001, Long-run Growth and Welfare Effects of Public Policies with Distortionary Taxation, Journal of Economic Dynamics and Control, 25, 2007-2042.

Barro, R. J., 1990, Government Spending in a Simple Model of Endogenous Growth, Journal of Political Economy, 98(5-2), S103-S125.

Bayoumi, T., M. Goldstein, y G. Woglom, 1995, Do Credit Markets Discipline Sovereign Borrowers? Evidence from U.S. States, Journal of Money, Credit, and Banking, vol. 27(41) (Noviembre), 1046-1059.

Chari, V. V. y P. J. Kehoe, 1998, On the Need for Fiscal Constraints in a Monetary Union, Federal Reserve Bank of Minneapolis Working Paper 589, (Agosto).

Glomm, G. y B. Ravikumar, 1994, Public Investment in Infrastructure in a Simple Growth Model, Journal of Economic Dynamics and Control, 18, 1173-1187.

King, R. G., C. I. Plosser, y S. Rebelo, 1988, Production, Growth and the Business Cycles. II. New Directions, Journal of Monetary Economics, 21, 309-341.

Lucas, R. E. Jr., 1987, Models of Business Cycles, Blackwell New York.

- 1990, Supply-side Economics: An Analytical Review, Oxford Economic Papers, 42, 293-316.

Rebelo, S., 1991, Long-run Policy Analysis and Long-run Growth, Journal of Political Economy, 99(3), 500-521.

Romer, P. M., 1986, Increasing Returns and Long-run Growth, Journal of Political Economy, 94(5), 1002-1037.

Stokey, N. L. y S. Rebelo, 1995, Growth Effects of Flat-rate Taxes, Journal of Political Economy, 103(3), 519-550.

Uctum, M. y M. Wickens, 1997, Debt and Deficit Ceilings, and Sustainability of Fiscal Policies: An Intertemporal Analysis, CEPR Discussion Paper, 1612. 
